\title{
Humidity sensor based on optical fiber coated with agarose gel
}

Susana Novais, Marta S. Ferreira, João L. Pinto

Susana Novais, Marta S. Ferreira, João L. Pinto, "Humidity sensor based on optical fiber coated with agarose gel," Proc. SPIE 11028, Optical Sensors 2019, 110281K (11 April 2019); doi: 10.1117/12.2520572

SPIE. Event: SPIE Optics + Optoelectronics, 2019, Prague, Czech Republic 


\title{
Humidity sensor based on optical fiber coated with agarose gel
}

\author{
Susana Novais*a $^{* a}$, Marta. S. Ferreira ${ }^{a}$, João. L. Pinto ${ }^{a}$ \\ ${ }^{a}$ I3N \& Physics Department, University of Aveiro, Campus de Santiago, 3810-193 Aveiro, Portugal.
}

\begin{abstract}
A reflective fiber optic sensor based on multimode interference for the measurement of relative humidity (RH) is proposed and experimentally demonstrated. The proposed probe is fabricated by fusion-splicing, approximately $30 \mathrm{~mm}$ long coreless fiber section to a single mode fiber. A hydrophilic agarose gel is coated on the coreless fiber, using the dip coating technique. When the incident light comes from the SMF to the CSF, the high-order modes are excited and propagate within the CSF. These excited modes interfere with one another as they propagate along whole CSF length, giving rise to a multimode interference (MMI). Since the effective refractive index of the agarose gel changes with the ambient relative humidity, as the environmental refractive index changes, the propagation constants for each guided mode within the CSF will change too, which leads to shifts in the output spectra. The proposed sensor has a great potential in real time RH monitoring, exhibiting a large range of operation with good stability. For RH variations in the range between $60 \% \mathrm{RH}$ and $98.5 \% \mathrm{RH}$, the sensor presents a maximum sensitivity of $44.2 \mathrm{pm} / \% \mathrm{RH}$, and taking in consideration the interrogation system, a resolution of $1.1 \% \mathrm{RH}$ is acquired. This sensor can be of interest for applications where a control of high levels of relative humidity is required.
\end{abstract}

Keywords: Fiber optic sensors; multimode interferometry; coreless fiber; relative humidity; agarose gel.

\section{INTRODUCTION}

Fiber optic sensing of relative humidity $(\mathrm{RH})$ has been extensively studied and there have been a great number of proposed methods. The measurement of RH has shown significant importance in a large range of applications, such as bacterial growth, process control, product quality, food and beverage processing, automotive, and meteorological industries [1]. Among various sensing methods of relative humidity, fiber optic based ones is preferred in many situations because of their minimal size and low weight, immunity to electromagnetic interference, corrosion resistance and remote sensing capability [2]. Though a large number of sensing schemes have been reported in literature, there is still a need for low-cost sensors, including both the fabrication and operation cost.

There are basically two operating mechanisms for the fabrication of fiber optic RH sensors. One is based on using specific technologies to form porous sensing structures, such as sputtering [3], electro-spinning [4], electron evaporation [5], or layer-by-layer nano-assembly [6]. The other type of RH fiber optic sensors involves using humidity sensitive coatings or gels (hydrophilic materials) on the surface or end face of the optical fiber, such as polyvinyl alcohol [7], polyethylene glycol [8], chitosan [9], polyethylene oxide [10] and agar [11]. This kind of polymer coatings has advantages of good performance [12], reproducibility, and long-term stability [10]. The hydrophilic materials normally swell physically and experience a refractive index change in response to change in RH.

Different fiber sensing structures have been combined with polymer coatings. For instance, the use of fiber Bragg gratings [13], photonic crystal fibers [14], Fabry-Perot cavities [15], Sagnac interferometers [16] and single-mode hetero-core fibers [10] have been proposed to detect environmental RH variations.

*novais@ua.pt; phone+351234370 200; fax +351234370 985.

Optical Sensors 2019, edited by Francesco Baldini, Jiri Homola, Robert A. Lieberman, Proc. of SPIE

Vol. 11028, 110281K · C 2019 SPIE · CCC code: 0277-786X/19/\$18 · doi: $10.1117 / 12.2520572$ 
Agarose is considered a biopolymer, originated from a marine alga (Gracilaria Verrucosa), whose use is commonly extended in the field of biochemistry for separation of DNA chains [17]. The agarose gel is considered a material with desirable humid sensitivity, able to readily absorb and desorb water, and to restore a fast equilibrium with atmospheric humidity. Besides that, it is extremely stable, not soluble in water and can be easily handled for device manufacture [13, 17].

This paper reports a fiber optic humidity sensor which is based on hydrogel coated optical fiber. The sensor is easy to produce, presents good resolution, particularly for environments with values of RH higher than $60.0 \% \mathrm{RH}$.

\section{SENSOR AND OPERATION PRINCIPLE}

The sensing device whose scheme is shown in Figure 1, involved two steps. The first step consisted on splicing a short section of coreless silica fiber (CSF) to single mode fiber (SMF), using the manual mode program of the splicing machine. The second step consisted in functionalizing the sensor with agarose gel, through dip coating technique.

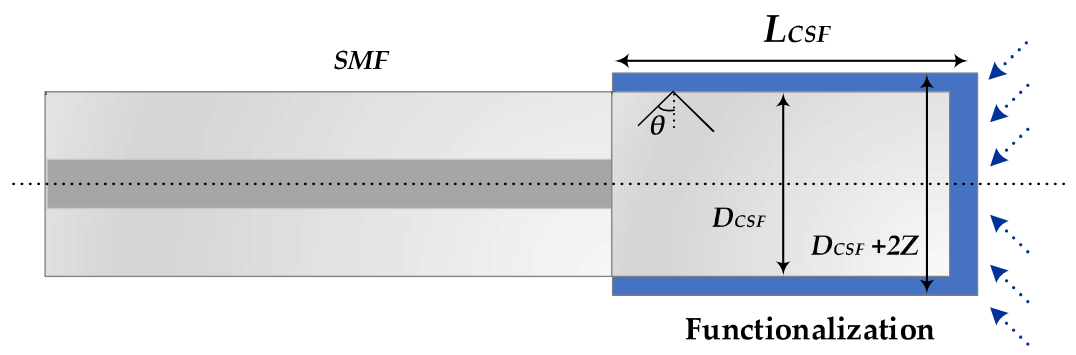

Figure 1. Schematic diagram of the sensor structure.

When the light guided though the SMF enters the CSF, the high-order modes are excited and propagate within the CSF. These excited modes interfere with one another as they propagate along whole CSF length, giving rise to a multimode interference (MMI). The reflected wavelength, $\lambda_{0}$, can be tailored according to Equation $1[18]$ :

$$
\lambda_{0}=\frac{n_{1} D_{C S F}^{2}}{2 L_{C S F}} p,
$$

where $n_{1}$, where $D_{C S F}, L_{C S F}$, and $p$ are the coreless fiber refractive index, the fiber diameter, the length, and the interference order number, respectively. The length and diameter of the CSF used in this work were $\sim 30 \mathrm{~mm}$ and $125 \mu \mathrm{m}$, respectively. Assumed the sensor dimensions in the present work, the refractive index (1.444) and the reflected wavelength in air $(\sim 1530) p$ was estimated to be 4. Taking into account Equation 1, there is no apparent dependence of the wavelength with the external medium. Nevertheless, if one considers the evanescent field produced at the CSF/external medium interface, the diameter can be considered as an effective value of $D_{C S F}+2 Z$, where $Z$ is the penetration depth. This parameter can be given by Equation 2 [19]:

$$
Z=\frac{\lambda_{0}}{2 \pi n_{1} \sqrt{\sin ^{2} \theta-\left(n_{s} / n_{1}\right)^{2}}},
$$

where $n_{s}$ is the surrounding medium refractive index and $\theta$ is the incident angle at the CSF/surrounding medium interface, as shown in Figure 1. Since the effective refractive index of the agarose gel changes with the ambient relative humidity, as the environmental refractive index changes, the propagation constants for each guided mode within the CSF will change too, which leads to shifts in the output spectra [20]. 


\subsection{Agarose gel coating}

The agarose solution was prepared by dissolving $0.9 \mathrm{~g}$ of agarose in $60 \mathrm{~mL}$ of distilled water, corresponding to a proportion of $1.5 \mathrm{wt} \%$. The solution was then heated up to $65^{\circ} \mathrm{C}$ and a magnetic stirrer was used to dissolve the agarose in distilled water. The sensing head was inserted in a silica capillary to ensure that the fiber was straight and stable (Figure 2a). The sensor was dipped into the hot agarose solution and pulled out very fast, by moving the horizontal platform downwards. When the agarose solution cools down and reaches room temperature, it polymerizes to form hydrogel and will not assume a liquid form again unless it is heated above the melting point. The coated sensor was left to dry for 48 hours at room temperature.

The agarose curing process was monitored with an optical interrogator (Figure 2a). The response was obtained in a spectral range between 1530 and $1570 \mathrm{~nm}$, immediately after removing the sensor from the agarose solution. This experiment was carried out in a controlled environment, with a room temperature of $25^{\circ} \mathrm{C}$. As shown in the Figure $2 \mathrm{~b}$, there is a higher wavelength shift in the first five minutes. From that moment on until 30 minutes, there is a smaller wavelength variation, which tends to stabilize after that time.

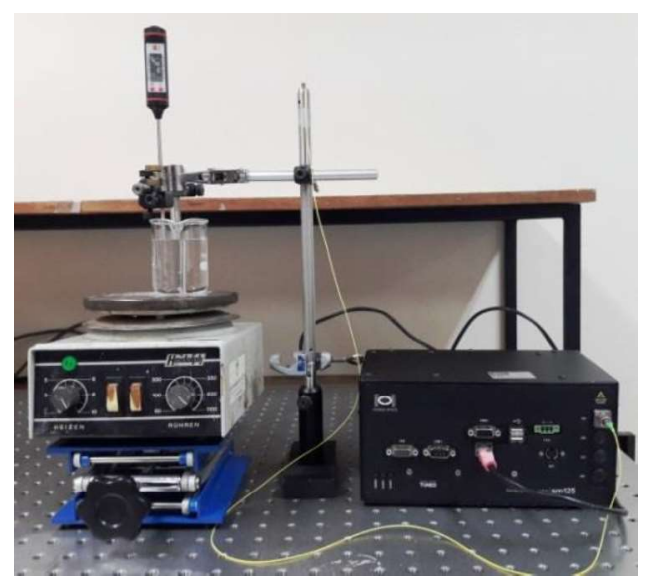

(a)

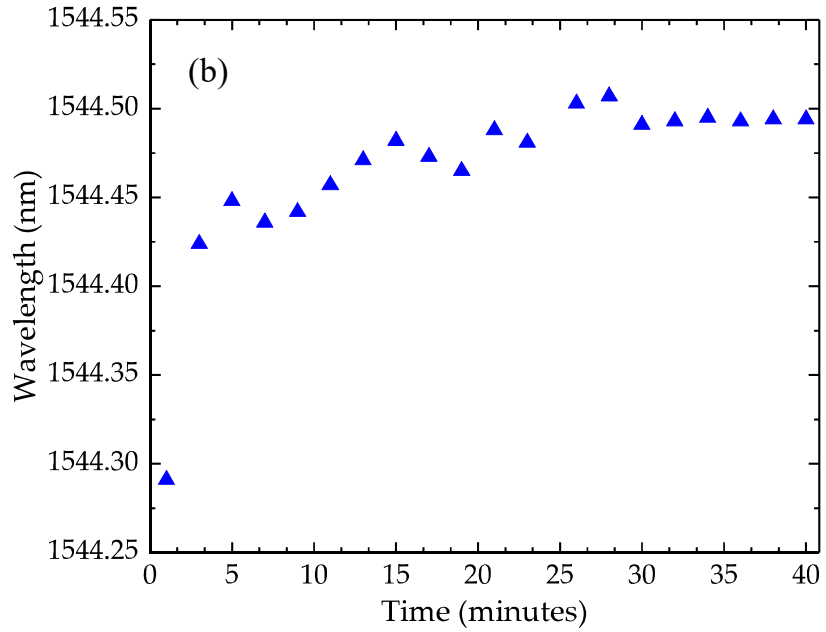

Figure 2. Photograph of the experimental setup for agarose deposition (a) and peak wavelength dependence with time during the agarose curing process (b).

\section{EXPERIMENTAL INVESTIGATION AND DISCUSSION}

Figure 3 displays the experimental setup used in this work for the characterization of the sensors. Two sensing structure, one uncoated and the other coated with agarose gel, with similar lengths $(28.8 \mathrm{~mm})$, were introduced in a thermal chamber, and subjected simultaneously to variations of relative humidity and temperature, ensuring that the sensors were exposed to the same environmental changes. This thermal chamber is equipped with a humidification-dehumidification system and a cooling-heating system that can change both RH and temperature in a controlled way. The optical fiber connector was preserved outside the thermal chamber and the reflection spectra were monitored using the interrogator sm125-500, Micron Optics Inc., operating at $2.0 \mathrm{~Hz}$ and wavelength accuracy of $1.0 \mathrm{pm}$. The RH experiments were carried out by keeping the temperature constant at $25{ }^{\circ} \mathrm{C}$ and by varying the $\mathrm{RH}$ between $20.0 \%$ and $98.5 \% \mathrm{RH}$, while the temperature measurements were done with a constant $\mathrm{RH}$ of $60.0 \% \mathrm{RH}$ and a temperature variation between $10{ }^{\circ} \mathrm{C}$ and $70^{\circ} \mathrm{C}$. After each change in the parameters, a period of 30 minutes was allowed for the thermal chamber to stabilize. 


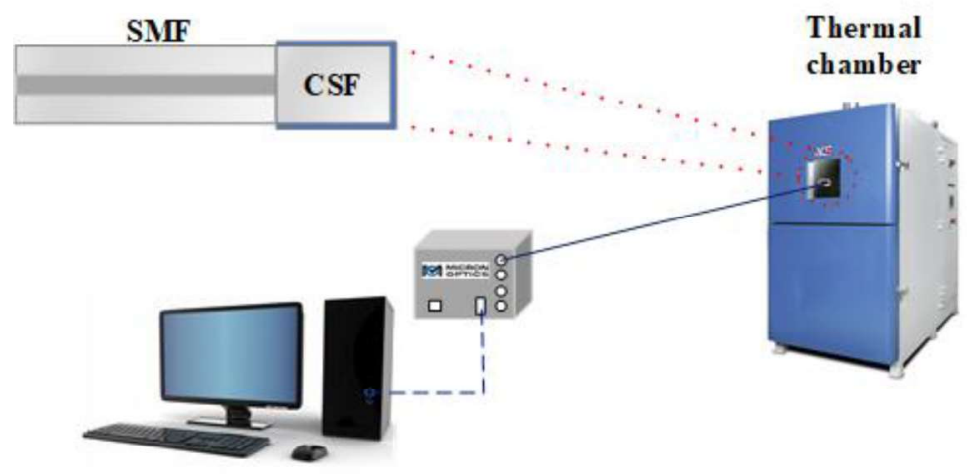

Figure 3. Experimental setup for the characterization of the sensors.

The Figure 4 (a) an (b) displays the wavelength shift dependence on the relative humidity and the temperature responses for both sensors respectively. As expected, the uncoated sensing head revealed to be insensitive to the RH variations. A linear fitting was adjusted to the experimental data, and a sensitivity of $0.9 \mathrm{pm} / \% \mathrm{RH}$ was attained. On the other hand, the coated sensor presented a non-linear wavelength shift towards longer wavelengths (red shift) with the external RH variations, which is more prominent for higher values of RH. This non-linear behavior has already been observed for this type of multimode interferometer sensors when subjected to refractive index variations [20], being related to the increase of the agarose gel refractive index with RH. Two different linear regions were considered to estimate the coated sensor sensitivity. The first region, for lower RH variations, ranges from $20.0 \%$ to $50.0 \% \mathrm{RH}$, whereas the second region, for higher RH variations ranges from $60.0 \%$ up to $98.5 \% \mathrm{RH}$. The sensitivities attained were of $18.6 \mathrm{pm} / \% \mathrm{RH}\left(r^{2}=0.988\right)$ and $44.2 \mathrm{pm} / \% \mathrm{RH}\left(r^{2}=0.922\right)$, for the first and second regions, respectively.
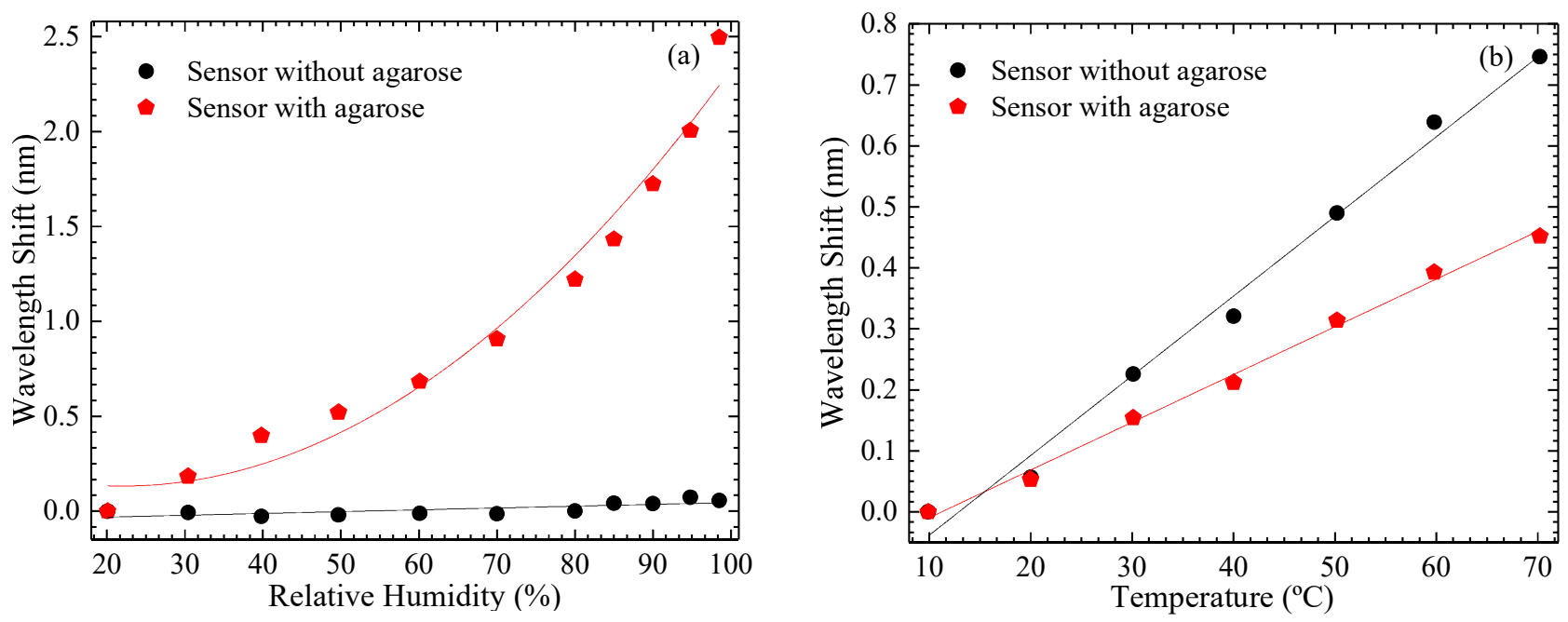

Figure 4. Measured wavelength shift dependence with RH (a) and temperature variations (b) considering both sensors.

The temperature responses of the sensors without and with agarose were measured using the same interrogation scheme as shown in Figure 3. The temperature was raised in steps of $10{ }^{\circ} \mathrm{C}$, from $10{ }^{\circ} \mathrm{C}$ up to $70{ }^{\circ} \mathrm{C}$ and $\mathrm{RH}$ was fixed to $60.0 \%$ RH. The response shown in Figure 4 (b) was linear in both cases. However, there was a drop in the sensitivity, from $13.6 \mathrm{pm} /{ }^{\circ} \mathrm{C}$ (uncoated sensor) to $7.8 \mathrm{pm} /{ }^{\circ} \mathrm{C}$ for the coated sensor. The sensor with agarose became approximately $2 \times$ less sensitive than the sensor without agarose. The cross-sensitivity between $\mathrm{RH}$ and temperature, for the coated sensor, was determined to be $0.2 \mathrm{RH} /{ }^{\circ} \mathrm{C}$.

The stability of the projected sensor was also experimentally explored. The coated sensor was placed in the thermal chamber at a constant $\mathrm{RH}$ and temperature of $60 \% \mathrm{RH}$ and $25{ }^{\circ} \mathrm{C}$, respectively. The peak wavelength was monitored 
during 30 minutes and the spectra were acquired once per minute. The same procedure was followed for a RH of $70 \%$ RH. The results are shown in Figure 5. For the first step, a mean wavelength of $1545.08 \mathrm{~nm}$ was determined, with a standard deviation of $13 \mathrm{pm}$, concerning the second step, the mean wavelength was of $1545.29 \mathrm{~nm}$, with a standard deviation of $12 \mathrm{pm}$. It should be highlighted that during the experiment, a temperature oscillation of $0.3{ }^{\circ} \mathrm{C}$ occurred in the thermal chamber, which might have also influenced the sensor response.

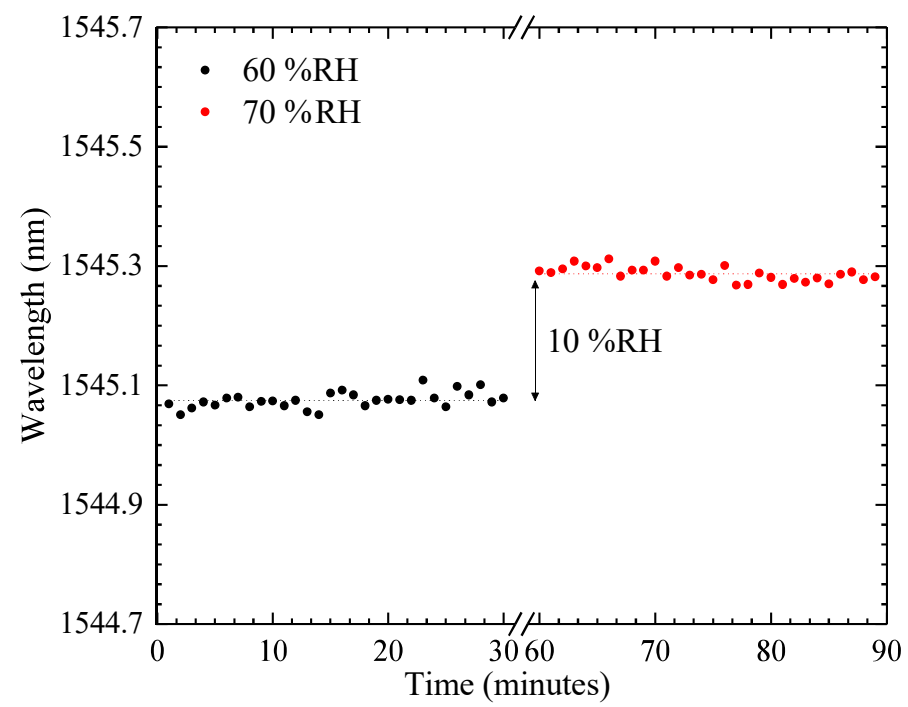

Figure 5. Step technique to estimate the resolution of the humidity sensor.

The minimum value of humidity, $\delta_{R H}$ that the sensor is able to discriminate is given by Equation 3 [21]:

$$
\delta_{R H}=2 \frac{\sigma_{\lambda} \Delta R H}{\Delta \lambda},
$$

where $\sigma_{\lambda}$ is the maximum standard deviation of $\lambda$ for both values of $\mathrm{RH}$, and $\Delta R H$ and $\Delta \lambda$ are the variation of $\mathrm{RH}$ and the mean wavelength shift between the two steps, respectively. By applying Equation 3, a resolution of $1.1 \% \mathrm{RH}$ was obtained. It is important to note that this value is greatly influenced by the spectral resolution of the equipment used for data acquisition.

\section{CONCLUSIONS}

In summary, a reflective optical fiber sensor based on coreless silica fiber (CSF) coated with hydrophilic agarose gel was proposed for the measurement of relative humidity $(\mathrm{RH})$ variations. For comparison purposes, both an uncoated sensing structure and a coated one were subjected to the same experiments. As expected, the uncoated sensor was insensitive to the environmental RH changes. On the other hand, the sensor with agarose gel exhibited a maximum sensitivity of $44.2 \mathrm{pm} / \% \mathrm{RH}$, with a resolution of $1.1 \% \mathrm{RH}$. The proposed sensor has a great potential in real time RH monitoring, particularly in environments with high percentages of humidity. One example of such applications are the lodges where Madeira wine is stored for ageing, where the RH is usually in the range between $65 \%-75 \% \mathrm{RH}$ (where the environment should be carefully controlled to ensure the desired quality of the product. The proposed sensor presented good stability, low manufacturing cost and easy fabrication, being an alternative to other sensors.

\section{ACKNOWLEDGEMENTS}

This work was financed by ERDF funds through the Regional Operational Program of the Center and National Funds through FCT (Fundação para a Ciência e Tecnologia) under the AROMA project (CENTRO-01-0145-FEDER-031568). 
Susana Novais gratefully acknowledges the funding of Project POCI-01-0145-FEDER-016414 PAC, cofinanced by Programa Operacional Competitividade e Internacionalização and Programa Operacional Regional de Lisboa, through the COMPETE 2020 Programme and National Funds through FCT for research fellowship BI/UI96/6643/2018. Marta S. Ferreira is also grateful for the research fellowship SFRH/BPD/124549/2016.

\section{REFERENCES}

[1] Torres, D. L., Elousa, C., Villatoro, J., Zubiac, J., Rothhardt, M., Schuster, K., Arregui, F. J., "Enhancing sensitivity of photonic crystal fiber interferometric humidity sensor by the thickness of $\mathrm{SnO} 2$ thin films," Sens. Actuators B: Chem 251, 1059-1067 (2017).

[2] Zhao, Z., Duan, Y., "A low cost fiber-optic humidity sensor based on silica sol-gel film," Sens. Actuators B: Chem 160, 1340-1345 (2011).

[3] Sigmund, P., "Elements of sputtering theory, in nanofabrication by ion-beam sputtering: Fundamentals and applications," Singapore: Pan Stanford, 1-40 (2012).

[4] Urrutia, A., Goicoechea, J., Rivero, P. J., Matías, I. R., Arregui, F. J., "Electrospun nanofiber mats for evanescent optical fiber sensors," Sens. Actuators B: Chem 176, 569-576 (2013).

[5] Yang, M., Xie, W., Dai, Y., Lee, D., Dai, J., Zhang, Y., Zhuang, Z., "Dielectric multilayer-based fiber optic sensor enabling simultaneous measurement of humidity and temperature," Opt. Express 22, 11892-11899 (2014).

[6] Urrutia, A., Goicoechea, J., Ricchiuti, A. L., Barrera, D., Sals, S., Arregui, F. J., "Simultaneous measurement of humidity and temperature based on a partially coated optical fiber long period grating," Sens. Actuators B: Chem 227, 135-141 (2016).

[7] Sun, H., Yang, Z., Zhou, L., Liu, N., Gang, T., Qiao, X., Hu, M., "A relative humidity sensing probe based on etched thin-core fiber coated with polyvinyl alcohol," Opt. Commun 356, 556-559 (2015).

[8] Acikgoz, S., Bilen, B., Demir, M. M., Menceloglu, Y. Z., Skarlatos, Y., Aktas, G., Inci, M. N., "Use of polyethylene glycol coatings for optical fiber humidity sensing," Opt. Rev. 15, 84-90 (2008).

[9] Voznesenskiy, S. S., Sergeev, A. A., Mironenko, A. Y., Bratskaya, S. Y., Kulchin, Y. N., "Integrated-optical sensors based on chitosan waveguide films for relative humidity measurements," Sens. Actuators B: Chem 188, no. 11, 482-487 (2013).

[10] Wu, Q., Semennova, Y., Mathew, J., Wang, P., Farrell, G., "Humidity sensor based on a single mode heterocore fiber structure," Opt. Lett. 36, 1752-1754 (2011).

[11] Huang, C., Xie, W., Yang, M., Dai, J., Zhang, B., "Optical Fiber Fabry-Perot Humidity Sensor Based on Porous Al2O3 Film," IEEE Photonics Technol. Lett. 27, 2127-2130 (2015).

[12] Bariáin, C., Matías, I. R., Arregui, F. J., López-Amo, M., "Optical fiber humidity sensor based on a tapered fiber coated with agarose gel," Sens. Actuators B: Chem 69, 127-131 (2000).

[13] Lee, C. L., You, Y. W., Dai, J. H., Hsu, J. M., Horng, J. S., "Hygroscopic polymer microcavity fiber Fizeau interferometer incorporating a fiber Bragg grating for simultaneously sensing humidity and temperature," Sens. Actuators B: Chem 222, 339-346 (2016).

[14] Mathew, J., Semenova, Y., Farrell, G., "Experimental demonstration of a high-sensitivity humidity sensor based on an Agarose-coated transmission-type photonic crystal fiber interferometer," Appl. Opt. 52, 3884-3890 (2013).

[15] Consales, M., Buosciolo, A., Breglio, G., Irace, A., Buontempo, S., Petagna, P., Giordano, M., Cusano, A., "Fiber optic humidity sensors for high energy physics applications at CERN," Sens. Actuators B: Chem 159, 66-74 (2011).

[16] Chen, L. H., Chan, C. C., Li, T., Shaillender, M., Neu, B., Balamurali, P., Menon, R., Zu, P., Ang, X., Wong, W. C., Poh, L. C., Leong, C. K., "Chitosan-coated polarization maintaining fiber based Sagnac interferometer for relative humidity measurement," IEEE J. Sel. Topics Quantum Electron 18, 1597-1604 (2012).

[17] Xu, W., Shi, J., Yang, X., Xu, D., Rong, F., Zhao, J., Yao, J., "Relative humidity sensor based on no-core fiber coated by agarose-gel film," Sensors 17, 2353 (2017).

[18] Lopez, J. E., Guzman, A., Arrioja, D. A., Aguilar, R., LiKamWa, P., "Tunable multimode interference bandpass fiber filter," Opt. Lett. 35, 324-326 (2010). 
[19] Fukano, H., Kushida, Y., Taue, S., "Sensitivity improvement of optical-fiber temperature sensor with solid cladding material based on multimode interference," Jap. J. Appl. Phys. 54 (2015).

[20] Novais, S., Ferreira, M. S., Pinto, J. L., "Optical fiber tip sensor for the measurement of glucose aqueous solutions," Photonics Journal, 10 (2018).

[21] Romero, R., Frazão, O., Pereira, D. A., Salgado, H. M., Araujo, F. M.; Ferreira, L. A., "Intensity-referenced and temperature-independent curvature-sensing concept based on chirped fiber Bragg gratings," Appl. Optics, 44, $3821-3826$ (2005). 